\title{
ONTOLOGY-DRIVEN SERVICE INTEGRATION INTO WEB APPLICATIONS: A DECLARATIVE APPROACH
}

\author{
Andreas Both ${ }^{1}$, Didier Cherix ${ }^{2}$ and Michael Martin ${ }^{3}$ \\ ${ }^{1}$ Anhalt University of Applied Science; DATEV eG, Köthen, Germany; Nuremberg, Germany \\ ${ }^{2}$ dokSAFE GmbH, Leipzig, Germany \\ ${ }^{3}$ Institute for Applied Informatics (InfAI), Leipzig, Germany
}

\begin{abstract}
The majority of web applications nowadays are data-driven. However, that does not mean that all data is available while launching the respective application. While considering Web 2.0 applications, data is often fetched on-demand from remote web services, for example, after a location was provided, weather data could be fetched, and local news is loaded. This mashup approach is highly dynamical, i.e., based on the data input of the user, completely different execution paths might be performed. Currently, such workflows are implemented within the application logic requiring high development effort and maintenance of the implemented logic to prevent unintentional behavior.

In this paper, we present a novel approach to integrate web services dynamically, to decrease deployment and maintenance costs and to enable the next generation of interlinked data web applications to enables application architects to (re)define the data integration in a descriptive way in an ontology, validate the workflows and define logical requirements. However, our approach is not just a design method but also a method for ad hoc integration of new services. Our approach has a significant impact on the effort for generating and maintaining dynamic applications.
\end{abstract}

\section{KEYWORDS}

Service Composition, Web Service Integration, Web Application Engineering, Ontologies

\section{INTRODUCTION}

In the era of the web, an ecosystem of services has been established which provides various functionality to the web community. Nowadays, web applications are widely used and often driven by the data. Web applications take advantage of (a) data often provided by such services, (b) data which not exists while web applications are released, and (c) data which is not self-maintained and respective data structure could change during the lifecycle of the processing web application. Additionally, it can be observed that varying services are integrated having the same purpose but different preconditions before they can provide data. Hence, using web services provide several benefits and are legitimately used widely to develop web applications.

However, dynamically integrated services might lead to side effects because of many different application execution paths resulting in application workflows that can only be predicted and controlled by investing much time. While integrating services, the application logic needs to be updated or changed to prevent unwanted (or unsafe) situations (e.g., do not make functionality available without having the corresponding preconditions fulfilled). There would be a great benefit in providing a mechanism for auto-wiring web services as it will increase the efficiency and safety of the service integration dramatically. In particular, the manual investments (e.g., by application architects) for the service integration with relation to the given data structures can be replaced by an automatic configuration. In contrast to tool-centered approaches to integrate services, our approach is data-driven and provide solutions for most of the data integration challenges.

We provide a methodology based on the Web Ontology Language (OWL) to describe the data model of the web application and its service interfaces. Using this vocabulary application architects can define preconditions of service calls and resulting data flows of the application. As a consequence, (a) less source code has to be created (less time to market and decreased maintenance efforts) and (b) flexible configurations are available in a descriptive format (integration of new resp. modification of existing services at runtime) as well as (c) improved agile development processes will be enabled (coupling, rearrangement and reuse of software components). Hence, our approach has a major impact on the generation, extension and maintenance of web applications that are driven by data provided by external services. 


\section{RELATED WORK}

A number of platform-specific frameworks emerged to assist ad hoc integration of functionalities into (web) applications, e.g., OSGi Service Platform ${ }^{1}$. Such frameworks ease development of modular and extensible applications, the control flow and integration logic still must be defined at design-time. In contrast, our approach enables emerging control-flows and specification of integration constraints at run-time.

In Web Engineering several methods have been proposed to componentize applications and, thus, make their development and evolution more efficient, e.g., WebComposition (Gellersen, 1997). Mashup platforms such as OMELETTE (Chudnovskyy, 2012) define coarse-grained components called widgets and enable ad hoc composition of applications by non-programmers. CRUISE (Pietschmann, 2011) operates with universal components such as data feeds, web services or UI widgets and applies Semantic Web technologies to perform run-time discovery and context-dependent adaptation of applications. However, this work focuses on data-driven components only and explores ways of their transparent integration into generic, also non-component-based applications. Hence, it is more general.

Intelligent integration of platform-independent modules has been discussed in the context of Semantic Web Services (SWS) aiming to enable automatic discovery, invocation and composition of distributed heterogeneous functionalities, e.g., OWL-S (Martin, 2004) and Minimal Service Model (Pedrinaci, 2010). Frameworks with automatic discovery and composition capabilities have been presented, e.g., proposing a method to semantically describe and compose web services, e.g. in (Rodriguez, 2015). However, the focus lies on application integration aspects and less on extensive capability modeling. Semantic technologies have been also applied to build generic web applications. Hera (Sluijs, 2010) enables the description of application structure and behavior using a set of semantic models and interpreted by a dedicated run-time engine. The presented approach also aims at defining business logic explicitly and in a declarative way. However, it doesn't require the whole application to be modelled semantically, but rather can be seamlessly integrated with in any environment and service type.

\section{BACKGROUND}

The Resource Description Framework $(\mathrm{RDF})^{2}$ is a general-purpose language for representing information in the web. On top of RDF the Web Ontology Language (OWL) is extending the vocabulary with more possibilities to describe relations between classes and properties. SPARQL is an RDF query language for adding, retrieving and changing RDF data. In the following the standardized Manchester syntax ${ }^{3}$ is used to represent RDF data. Here, besides standard properties like SubClassof and $r d f:$ label we use just the following expressions, where $A$ and $B$ are resources and prop is a property (c.f., Listing 1).

\begin{tabular}{l|l|l|}
$\begin{array}{l}\text { A prop: some B: If A and B are } \\
\text { resources, then the constraint hold iff } \\
\text { there are instances available, s.t., it } \\
\text { matches the expression A prop: B. }\end{array}$ & $\begin{array}{l}\text { A prop: max O B \}: If A and B are } \\
\text { resources, then the constraint hold iff } \\
\text { there is no data available matching the } \\
\text { expression A prop: B. }\end{array}$ & $\begin{array}{l}\text { A EquivalentTo: B } \\
\text { (symmetric relation): If A and } \\
\text { B are classes, then each } \\
\text { instance of A is also an instance } \\
\text { of B. }\end{array}$ \\
\hline
\end{tabular}

Listing 1. Used RDF expressions in Manchester syntax

For evaluation of constraints reasoners (reasoning engines) are used. They ensure that the defined rules are obeyed and highlight constraint violations (implementation depended). Here, we use only reasoners that follow the closed world assumptions, i.e., unless an atomic sentence is known to be true, it can be assumed to be false. In this paper we use the following (web) service definition: A call of a service $S$ is defined by the signature $R$ $S(P)$, where $R$ is a struct of return values and $P$ is a list of named parameters.

\footnotetext{
${ }^{1}$ https://www.osgi.org/developer/architecture/

${ }^{2}$ http://www.w3.org/TR/2004/REC-rdf-syntax-grammar-20040210/

${ }^{3}$ http://www.w3.org/TR/owl2-manchester-syntax/
} 


\section{PROBLEM DESCRIPTION AND GOALS}


Figure 1. Exemplary web portal and corresponding service signatures

Developing an application in the Web 2.0 era is very often driven by the integration of external services. In Figure 1 an application is described that allows to login (A) and shows thereafter the emails of the user (C). In addition, for the provided username a search is performed (D). The application also shows the weather of the user's location (B). All data is retrieved from external services that need to be integrated within the application. During the engineering process the application architect has to decide which functionality of external services has to be defined. E.g., username and password are used to identify the user and fetch the profile data of the user (it is also possible to login with an accesstoken), the emails of the user are part of the profile data, weather information is presented for the current user's location.

Application architects must capture all possible execution paths within the application. Therefore, we derive the following requirements: (R1) as application architect, I want to change the data flows of the application via configuration, and (R2) I want to integrate new services within the application via configuration, (R3) each service can be defined independently from the application including its constraints. Concluding, applications should integrate web services via a descriptive methodology.

\section{APPROACH AND EXECUTION PROCESS}

First the creation of the application data model $a d m$ needs to be performed while defining all available data types and data items while defining parameters, expected return values and the constraints. ${ }^{4}$

\begin{tabular}{|l|l|}
\hline Class: $\mathrm{n}$ & Prefix adm: <urn:example:current-application-data-model> \\
SubClassOf: gd:value some t, & Class: adm:IP \\
gd:value some gd:Thing & SubClassOf: gd:Decimal \\
DatatypeProperty: gd:value. & SubClassOf: gd:Thing \\
\hline
\end{tabular}

Listing 2. General definition of a data type and the exemplary definition of the IP class

A data type ${ }^{5}$ of the $a d m$ with the name $\mathrm{n}$ and the data type $t$ is defined as in Listing 3 . For example, a string data type can be defined with $n:=g d:$ String and $t:=x$ sd: string, making available a String data type within in the namespace $\mathrm{gd}$ and of type String of the xsd namespace which is also a subtype of

\footnotetext{
${ }^{4}$ A generalized exemplary implementation is shown in the online appendix using the services described in Figure 1 available at https://cutt.ly/CwTm75s.

${ }^{5}$ To be clear this definition of data type has nothing in common with RDF datatype.
} 
$\mathrm{gd}$ : Thing. ${ }^{6}$ Now, it is possible to define data items needed for the application as subtypes of the defined data types. A data item within the application data model $a d m$ with the name $\mathrm{n}$ and the data type $t$ is defined as shown in Figure 1. The functionality shown there might be implemented using the corresponding services. The goal of the application is to provide the information to the user depending on the data that was made available. Data can be made available via user input (username) or by the client (IP).

\begin{tabular}{l|l} 
(a) Class: $\mathrm{n}$ & (b) Class: adm:username
\end{tabular} SubClassOf: $\mathrm{t}$ SubClassOf: gd:String

Listing 3. General definition of a parameter and the exemplary definition of username

First, data items of the service are defined as resource. Data items of a service are made known by defining the label of each parameter. Additionally, for each field within the returned result the label is defined, too, as shown in Listing 4.

\begin{tabular}{|l|l|}
\hline $\begin{array}{c}\text { (a) Class: s:n } \\
\text { Annotations: rdfs:label "n" }\end{array}$ & (a) Class pp:password \\
Annotations: rdfs:label "password"
\end{tabular}

Listing 4. General definition of a return values and the exemplary definition of returning a password variable

Up to now the $a d m$ does not clarify whether the data item is a parameter of the service call or part of the result. It just defines the classes needed for the later data bindings. Hence, just the service data was defined.

Secondly, the preconditions of a service call have to be defined. In OWL syntax we use a type definition to represents that input parameters are necessary. A service is defined within the gd namespace and a relation called has Param is defined to express the bound of a service instance to input parameters (see Listing 5).

\begin{tabular}{|l|r|}
\hline (a) Class: gd:Service & (b) $\begin{array}{r}\text { Class se:constraint } \\
\text { SubClassOf gd:Service } \\
\text { ObjectProperty: gd:hasParam }\end{array}$ \\
$\begin{array}{r}\text { EquivalentTo: ( gd:hasParam some se:query ) } \\
\text { Domain: gd:Service }\end{array}$ & $\begin{array}{r}\text { and:hasParam max 0 se:searchresults ) } \\
\text { Range: gd:Thing }\end{array}$ \\
\hline
\end{tabular}

Listing 5. General definition of the input of a service and the exemplary constraint of the searchengine service (se)

Now, it is possible to define the precondition for a service call. For a given service $s$ (represented as namespace) a constraint is defined that demands that all mandatory parameter $p i$ are available and have at least one valid value assigned (in OWL: some) and at least one return value $\bar{r}_{-} j$ was not computed yet (i.e., is not available). See the following listing for the general definition and an example (c.f., Listing 6).

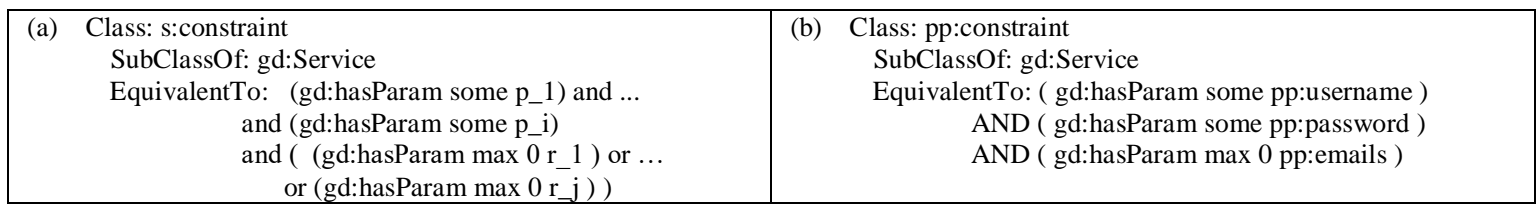

Listing 6. General definition of a service call precondition and the exemplary constraint of the pp service

Given these definitions of the services and their parameters, a formal representation of the service landscape was established within the constructed $a d m$. Note that this representation is compatible with any kind of real-world service description. Hence, the requirement of composability is fulfilled.

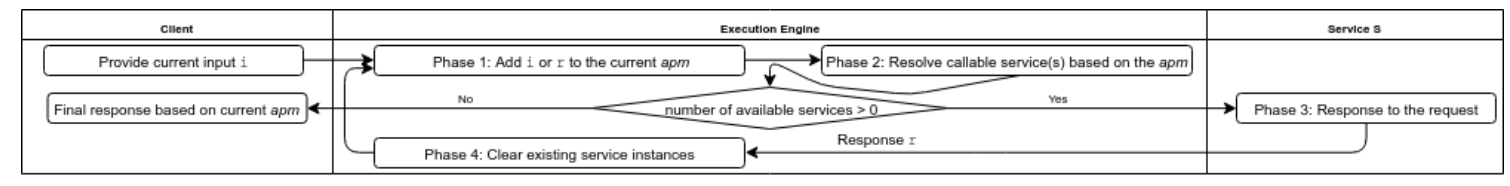

Figure 2. Execution process of automatic data integration 
After creating the initial $a d m$ it is used in an execution process consisting of the phases (c.f., Figure 2): (1) data assignment, (2) constraint evaluation, (3) the execution of service calls, and (4) closing model cleanup. In phase 1 (data assignment) the initial data application model $a d m$ is interpreted. Without any loss of generality, it is assumed that all data items of the $a d m$ are (defined but) not instantiated. To initialize a data flow, at least one data item of the apm must be instantiated. A new resource is inserted representing the value and making it available within the apm. Here, there is no constraint whether the data was assigned by a manual data input or any other interaction with the $a d m$. In phase 2 every constraint of the ontology is evaluated. This is done by just constructing a new service instances as the ontology will only allow to be instantiated if all corresponding constraints are obeyed. After this step the ontology contains instances of the type gd: Service that represent the services being enabled to be called. Thereafter all instances of services are available obeying the related preconditions. In phase 3 the execution engine queries all service types (i.e., the services that can be called) via SPARQL. One service is chosen to be called (concurrent calls are possible but avoided in this paper for the sake of clarification). The required input parameters to call the chosen service are fetched from the ontology. The response of called service is stored in a temporal variable $r$. In phase $\mathbf{4}$ all service instances are removed from the ontology. Thereafter, no service instances are available within the ontology. Now, the response data provided in $r$ is used within phase 1 to establish a process loop.

\section{CONCLUSION}

Nowadays, many web applications are data-driven. In many cases, the data is not available completely within the application but needs to be fetched from remote services (often called mashup). Hence, integrating the data of web services is one of the main tasks for architects designing and developing industrial and academic web applications. However, it causes serious and increasing development effort using traditional methodologies where the application data flow logic is implemented (manually) in program code.

The main contribution our approach is the methodology of integrating services automatically. Our approach is capable of using the provided data within the application and to control the constraints in a descriptive way. In contrast to previous work, the application workflow is driven by configuration only. Additionally, it can be updated and extended at runtime and is not fixed to one type of web services.

The described process engine is independent from the actual service implementations and just driven by the ontology. This is a key feature of our method as it provides a formal description of the services and the process. The ontology is also the key asset on the way to control the application behavior. While using a standard vocabulary for describing the constraints, standard components (i.e., reasoners) can be used for validating the preconditions of service calls. Finally, the control of the application is actually in the hands of the application architect, leading to a consistent documentation, clear constraints and implementation of the data workflows leading to an application data model that can be extended and validated.

In the future, we will extend the data model by additional functionalities, e.g. time dependency, a data flow evaluation process (to prevent unreachable services) and integrating interfaces version (Arndt, 2018).

\section{REFERENCES}

Arndt, N. et al., 2018. Decentralized collaborative knowledge management using git. Journal of Web Semantics.

Chudnovskyy, O. et al., 2012. End-User-Oriented Telco Mashups: The OMELETTE Approach. In WWW 2012 Companion Volume, pages 235-238.

Gellersen, H.-W. et al., 1997. WebComposition: an object-oriented support system for the web engineering lifecycle. Computer Networks and ISDN Systems, 29(813):1429 - 1437.

Martin, D. et al., 2004. OWL-S: Semantic markup for web services. W3C member submission, 22:2007-04.

Pedrinaci, C. and Domingue, J., 2010. Toward the next wave of services: Linked services for the web of data. J. of Universal Comp. Science, 16(13):1694-1719.

Pietschmann, S. et al., 2011. Semantics-based discovery, selection and mediation for presentation-oriented mashups. 5th International Workshop on Web APIs and Service Mashups, Mashups '11. ACM.

Rodriguez-Mier, P. et al., 2015. An Integrated Semantic Web Service Discovery and Composition Framework. IEEE Transactions on Services Computing, 9(4), pages 537-550. 